\title{
Unilateral increased transradiancy of the lung caused by bronchial carcinoid tumour
}

\author{
S A SPITZER, I SEGAL, E LUBIN, M NILI, AND M LEVY
}

From the Puimonary Unit, Department of Nuclear Medicine and Department of Cardiothoracic Surgery, Beilinson Medical Center, Petah Tiqva, Tel Aviv University, Sackler School of Medicine, Israel

ABSTRACT Two patients with an intraluminal carcinoid tumour obstructing the right main bronchus in one, and the left main bronchus in the other, resulting in unilateral increased transradiancy from hypoperfusion of the lung, are described. Resection of the tumours and reconstruction of bronchial continuity was followed by return of perfusion to normal between three and 18 months after the operation. The patient in whom the diagnosis was delayed showed a much slower rate of return of perfusion and a small lung resulted. The finding of increased transradiancy of lung even in an asymptomatic patient should be an indication for bronchoscopy.

The phenomenon of hypoperfusion of the lung causing increased transradiancy is rarely observed in bronchial carcinoid tumours. Hepper et al ${ }^{1}$ recently reported a case which was associated with the carcinoid syndrome and they reviewed previous publications. We have been able to investigate and make observations on the rate of the return of perfusion to the affected lung, after successful bronchial sleeve resection in two patients found to have a carcinoid tumour of a main bronchus with a ball-valve type of obstruction.

\section{Case reports}

\section{CASE 1}

A 34-year-old white woman, a non-smoker, was seen because of cough and expectoration of purulent sputum for 18 months and recurrent haemoptysis for six months. She was treated in a local chest clinic where a chest radiograph was normal, and a presumptive diagnosis of bronchiectasis with recurrent bronchitis was made. Her symptoms responded well to treatment with antibiotics. She denied wheezing, flushing, and shortness of breath, nor did she complain of any gastrointestinal symptoms.

On physical examination the chest was found to be symmetrical with good expansion and emptying. Breath sounds were markedly diminished over the right hemithorax. Heart and abdomen were not remarkable. The routine tests of urine, haemo- globin, leucocytes, sedimentation rate, and blood chemistry yielded normal values. The urinary excretion of 5-HIAA was within normal limits.

A radiograph (fig 1) showed increased transradiancy of the entire right lung caused by decreased vascular markings. The trachea was slightly shifted to the right. There was some fullness of the right hilar shadow. An expiratory film

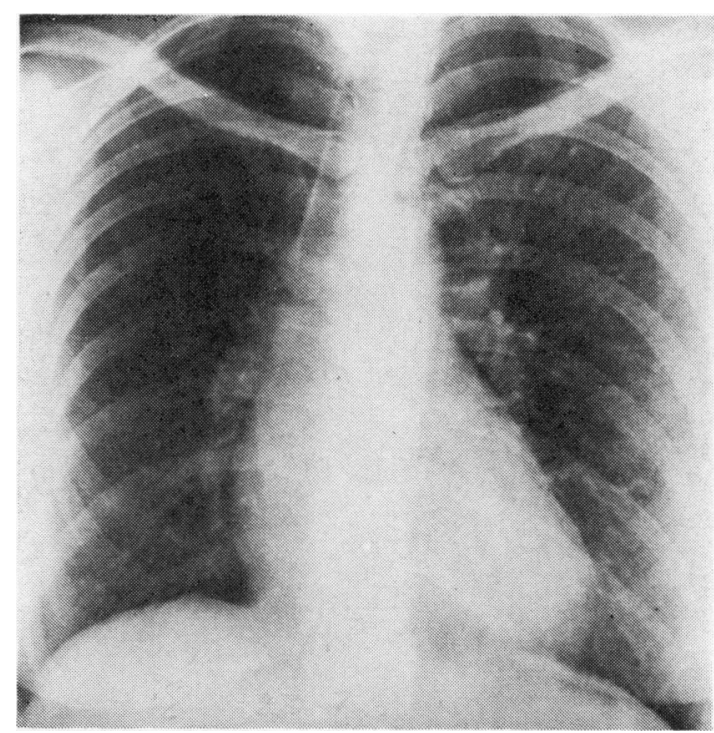

Fig 1 Case 1. Chest radiograph, inspiratory film. Increased transradiancy of right lung. 
(fig 2) demonstrated lack of emptying of the right lung and a shift of the mediastinum to the left.

A lung perfusion scan (fig 3 ) with radioiodinated macroaggregated albumin showed no perfusion of the right lung. Bronchoscopic examination showed a mobile pedunculated tumour, which bled easily, obstructing the orifice of the right main bronchus. Microscopic examination of a biopsy specimen revealed an adenoma of the carcinoid type.

Right thoracotomy revealed a markedly hyperinflated right lung. The tumour which was palpated within the right main bronchus was found to originate from the medial aspect of the bronchus and to obstruct almost the whole lumen. Resection of a triangular sleeve of the main bronchus was accomplished preserving all the lung parenchyma. Bronchial continuity was re-established by approximation of the cut ends of the bronchus. Examination of the resected specimen showed a polypoid bronchial carcinoid tumour 15 $\mathrm{mm}$ in diameter involving the full thickness of the membranous bronchial wall, with tumour-free margins at the lines of resection. Recovery from operation was uneventful. A radiograph of the thorax showed normal vascular markings in the right lung. Sequential lung scans after operation showed gradual normalisation of perfusion of the right lung, so that by the end of three months it was considered to be normal (fig 3).

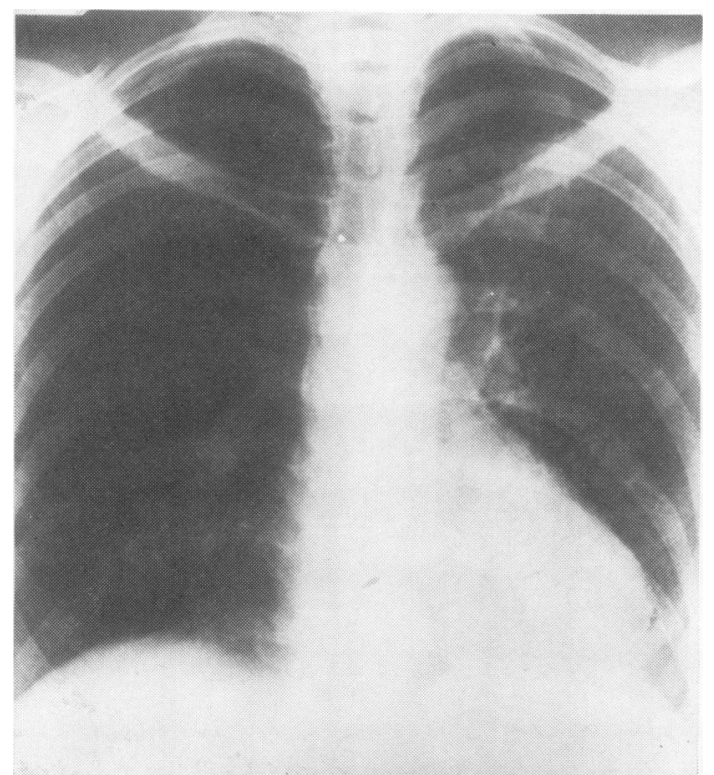

Fig 2 Case 1. Chest radiograph, expiratory film. Failure of emptying of right lung and shift of mediastinum to left.
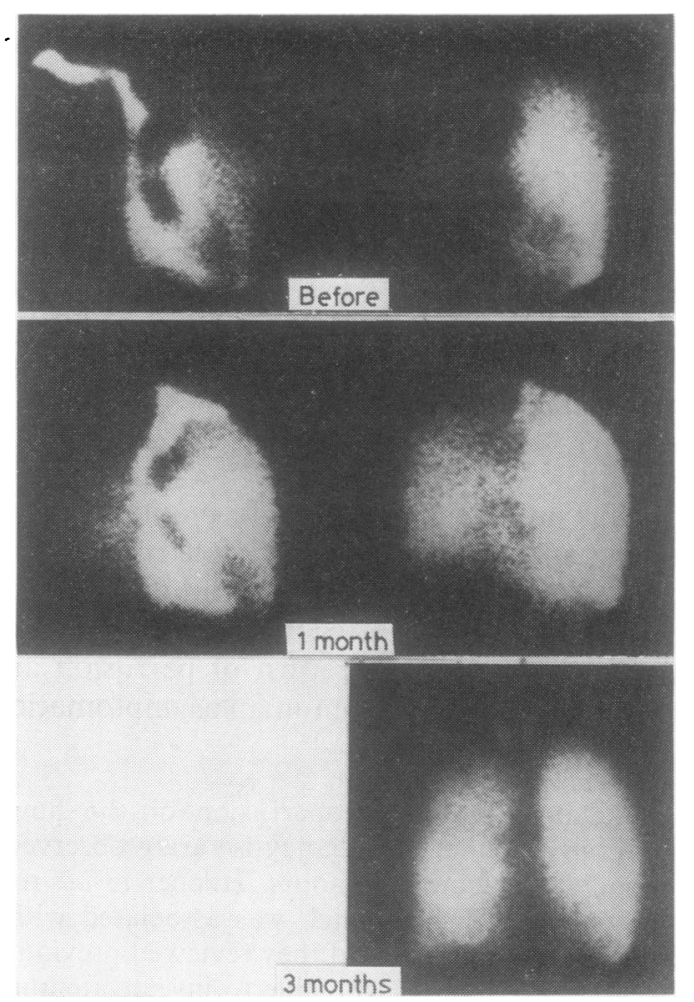

Fig 3 Case 1. Lung perfusion scan before (top), one (middle), and three (bottom) months after operation. The left hand scans represent early perfusion and the right hand late perfusion scans.

CASE 2

A 35-year-old white woman, a non-smoker, pre sented in the ninth month of her eighth pregnancy She was seen because of a cough productive of purulent sputum, dyspnoea on exertion, ande wheezing for six months, which followed a febriles illness. A course of ampicillin improved her symp을. toms but she continued to have a dry non-prot ductive cough. One day before admission she developed sharp retrosternal chest pain radiating to her back.

On physical examination she was found to be ato full term. The chest was symmetrical with mildlyo restricted expansion and slow emptying. Breath sounds were markedly diminished over the entire? left hemithorax, the heart was not remarkable, 7 and the abdomen was compatible with full term? pregnancy. Routine tests of urine, haemoglobin, $\stackrel{\mathbb{Q}}{\stackrel{Q}{ }}$ leucocytes, sedimentation rate, and blood chem- $\mathbb{\otimes}$ istry yielded normal values. The urinary excretiono of 5-HIAA was within normal limits.

A chest radiograph (fig 4) showed increased trans-oํㅇ 


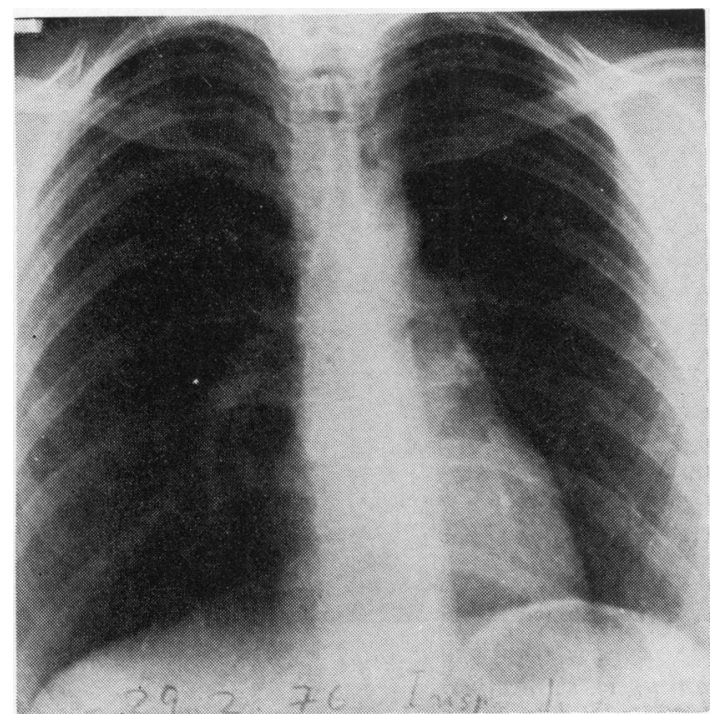

Fig 4 Case 2. Chest radiograph, inspiratory film. Increased transradiancy left lung.

radiancy of the entire left lung caused by decreased vascular markings. The left hilar shadow was normal. The patient returned three months later after giving birth to a normal child. The chest radiograph had not changed. An expiratory film (fig 5) demonstrated good emptying of the right and lack of emptying of the left lung with a marked shift of the mediastinum to the right side. A lung scan with ${ }^{99^{\mathrm{m}}}$ Technetium macroaggregated albumin (fig 6) showed no perfusion of the left lung. Pulmonary function tests demonstrated a mild restrictive and moderate obstructive ventilatory defect with air trapping and marked reduc-

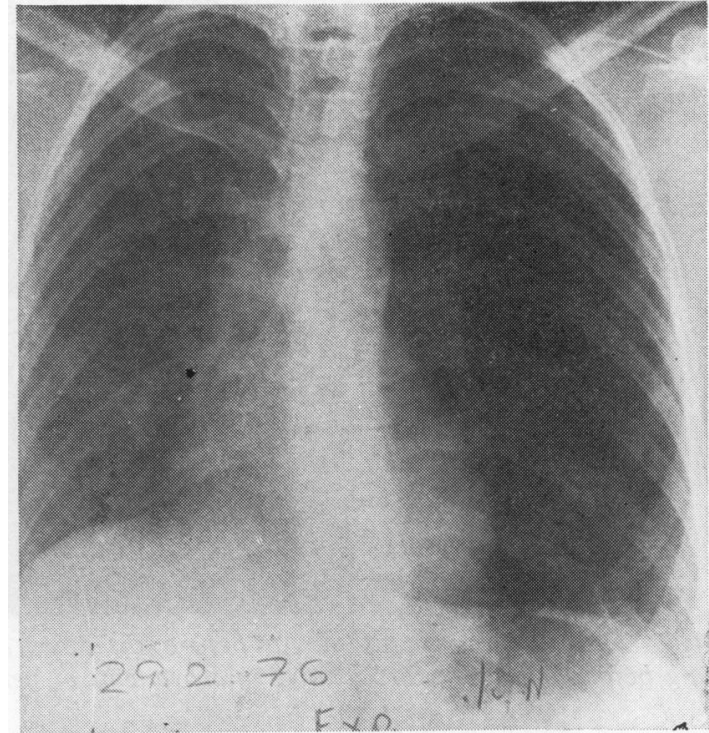

Fig 5 Case 2. Chest radiograph, expiratory film. Failure of emptying of left lung with shift of mediastinum to right.

tion in diffusing capacity. Arterial blood gases were normal (table).

The patient remained asymptomatic except for a mild cough and dyspnoea on exertion. One year later she returned with signs of a left-sided hydropneumothorax. A chest tube was inserted, and air and fluid drained. The lung, however, remained collapsed. A tomogram of the left hilum (fig 7) demonstrated a shadow protruding into the lumen of the terminal portion of the left main bronchus. Bronchoscopy revealed a tumour totally obstruct-

Table Lung function tests before and one year after operation in case 2

\begin{tabular}{|c|c|c|c|}
\hline Lung function test & Predicted & Before operation & One year after operation \\
\hline Vital capacity (1) & $3 \cdot 22$ & $2.38(74)$ & $2.44(74)$ \\
\hline $\begin{array}{l}\text { Functional residual capacity (l) } \\
\text { (helium dilution) }\end{array}$ & $2 \cdot 67$ & $2 \cdot 29(86)$ & $2 \cdot 17(81)$ \\
\hline $\begin{array}{l}\text { Thoracic gas volume (l) } \\
\text { (plethysmograph) }\end{array}$ & $2 \cdot 67$ & $3 \cdot 31(124)$ & $2 \cdot 72(101)$ \\
\hline Residual volume (1) & $1 \cdot 54$ & $1 \cdot 64(106)$ & $1 \cdot 35(87)$ \\
\hline Total lung capacity (1) & $4 \cdot 76$ & $4.02(84)(\mathrm{He})$ & $3.79(78)$ \\
\hline RV/TLC $(\%)$ & 31 & 41 & 35 \\
\hline $\mathrm{FEV}_{1}(\mathrm{l})$ & $2 \cdot 86$ & $1 \cdot 75(61)$ & $2 \cdot 0(70)$ \\
\hline $\mathrm{FEV}_{1} / \mathrm{VC}(\%)$ & 83 & 73 & 82 \\
\hline FEF $25 \%-75 \%(1 / s)$ & $3 \cdot 24$ & $1 \cdot 42(44)$ & $1 \cdot 73(53)$ \\
\hline Airway resistance $\left(\mathrm{cmH}_{2} \mathrm{O} / \mathrm{l} / \mathrm{s}\right)$ & $1 \cdot 24 \pm 0.5$ & $4 \cdot 21$ & $1 \cdot 72$ \\
\hline $\begin{array}{l}\text { Pulmonary diffusion capacity }(\%) \\
\text { (steady state) }\end{array}$ & $16 \cdot 8$ & $8 \cdot 4(50)$ & $13 \cdot 5(80)$ \\
\hline $\mathrm{PaO}_{2}(\mathrm{~mm} \mathrm{Hg})$ & $80-90$ & 85 & 91 \\
\hline $\mathrm{PaCO}_{2}(\mathrm{~mm} \mathrm{Hg})$ & $40 \pm 2$ & 30 & 34 \\
\hline pH (units) & $7.4 \pm 0.02$ & $7 \cdot 43$ & $7 \cdot 44$ \\
\hline
\end{tabular}

Figures in parentheses represent percentage of predicted. 


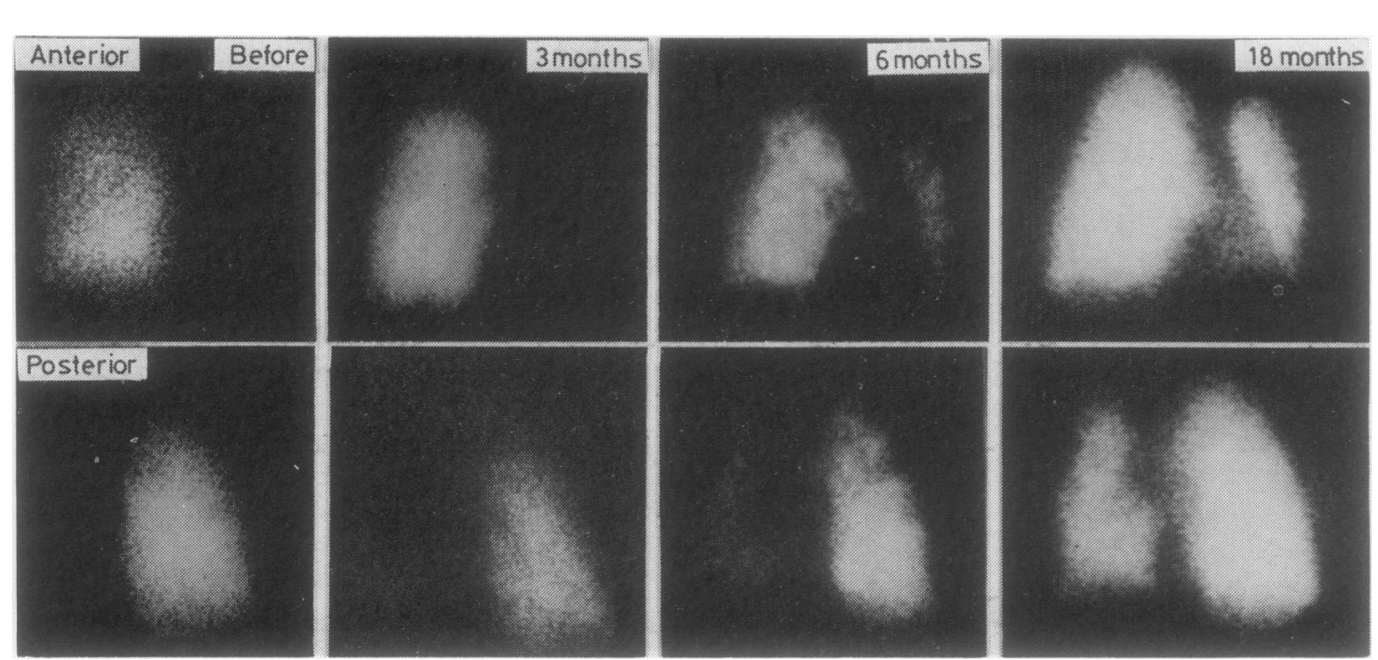

Fig 6 Case 2. Lung perfusion scan before, three, six, and 18 months after operation. No perfusion of left lung before operation. Progressive improvement of perfusion up to 18 months.

ing the left main bronchus just before the bifurcation. Microscopic examination of a biopsy specimen demonstrated an adenoma of the carcinoid type. Left thoracotomy revealed a collapsed lung. The tumour which was $2 \mathrm{~cm}$ in diameter was found to originate from the postero-lateral aspect

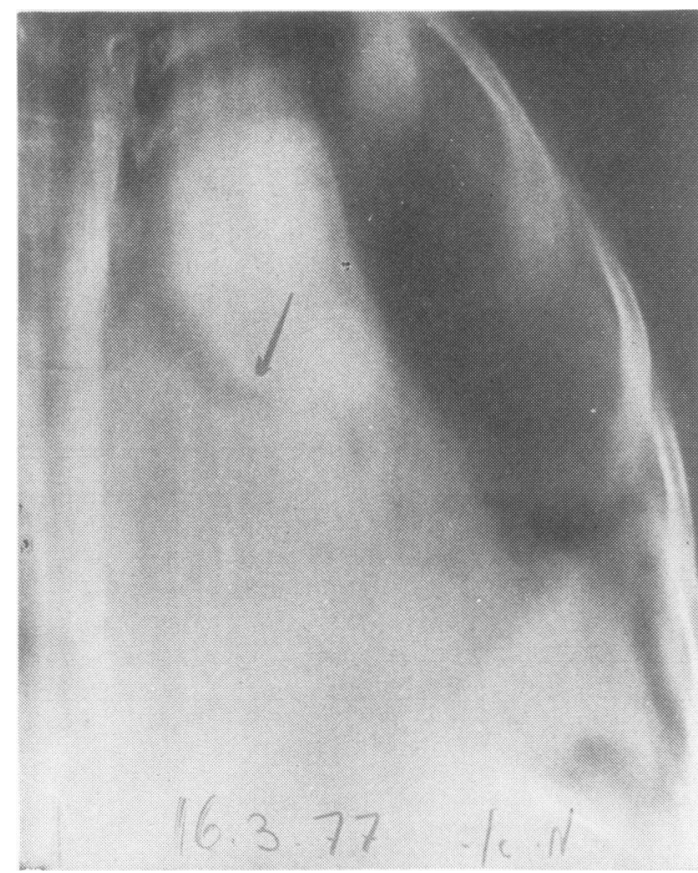

Fig 7 Case 2. Tomogram of left hilum, showing tumour obstructing left main bronchus. of the bronchus and to obstruct the whole lumen: A sleeve of the left main bronchus was resecteb and bronchial continuity was re-established by aps proximation of the cut ends. Examination of theo resected specimen showed a polypoid bronchiab̆ carcinoid tumour $20 \mathrm{~mm}$ in diameter involving the full thickness of the membranous bronchia 5 wall with tumour-free margins at the line of re- 3 section. Recovery from operation was uneventful A radiograph of the thorax showed a small lef? lung, with shift of the mediastinum to the left and an elevated left diaphragm (fig 8). SequentiaP

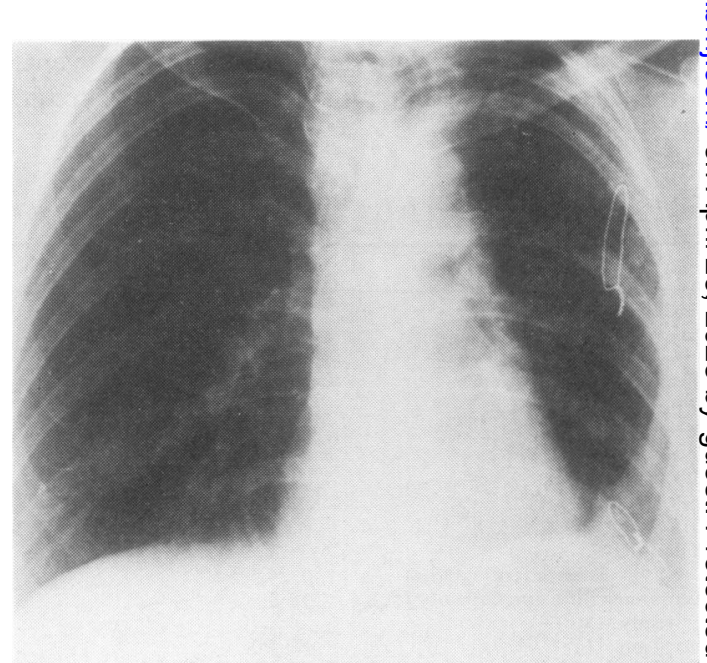

Fig. 8 Case 2. Chest radiograph after operation. Small left lung with mediastinal shift to left. 
lung scans (fig 6) performed three, six, and 18 months after operation showed gradual normalisation of perfusion of the left lung. By the end of six months, perfusion of the left lung was still reduced compared with the right. After 18 months the perfusion to the small left lung was considered to be normal. Pulmonary function tests performed one year after operation showed again a mild restrictive ventilatory defect but disappearance of air trapping. The obstructive ventilatory defect had improved as manifested by increase in flow rates and reduction in airway resistance. The diffusing capacity had improved considerably (table).

\section{Discussion}

The phenomenon of unilateral hypoperfusion of the lung secondary to a ball-valve mechanism partially obstructing a main bronchus is occasionally observed. In three surveys totalling 165 cases of bronchial adenoma, ${ }^{2-4}$ the standard chest radiograph showed an abnormal mass or collapse of a lung segment. In none of the survey patients did the chest radiograph show the phenomenon of increased transradiancy of the lung. Several isolated case reports describing the phenomenon of increased transradiancy of the lung in bronchial adenoma have been reported. ${ }^{5}$ This phenomenon is to be expected only where the adenoma shows a strictly intraluminal growth. Since this type of adenoma occurs in only $10 \%$ of the cases, ${ }^{7}$ its rarity can be explained.

Another reason for the rarity of this phenomenon is the fact that every intraluminal growth eventually causes total collapse of the corresponding lung segment as occurred in case 2. Delay in the diagnosis will miss that time period where the phenomenon of increased transradiancy was present.

The cause of increased transradiancy can be attributed clearly to the marked diminution or even total absence of perfusion of the lung. This has been clearly demonstrated in the perfusion scan in both our patients which showed no radioactivity over the involved lung. The causes for the hypoperfusion of the lung have been elucidated by the experimental work of von Euler and Liljestrand and others. ${ }^{8-11}$ They have demonstrated that alveolar hypoxaemia causes severe arterial vasoconstriction.

The longer alveolar hypoxaemia is present the more persistent the arterial vasoconstriction after removing the cause for the alveolar hypoxaemia. Although it is difficult to determine the moment when in the course of development of an adenoma the stage of alveolar hypoxaemia has started, we estimated on clinical grounds that this stage lasted approximately six months in the first patient and 24 months in the second. Accordingly the full restitution of perfusion in the first patient occurred within three months of operation while perfusion improved more slowly in the second patient and only reached normality 18 months after operation. This is an indication for early bronchoscopy in even asymptomatic patients with unilateral increased transradiancy of the lung. The result of the tests of pulmonary function may hint at the existence of a ball-valve type of bronchial obstruction. ${ }^{1}$ The pattern of a restrictive ventilatory defect with considerable air trapping estimated by body plethysmography, and markedly diminished transfer factor with normal gas exchange, should raise the suspicion of this condition. This pattern was demonstrated in our second patient and should have led to an earlier diagnosis of the disease.

It is assumed that the physiological parameters measured one year after surgical reconstruction have not reached normality, because of the reduction of the size of the left lung. The cause of the reduction in the size of the lung even though perfusion has been re-established is not known. A lung biopsy could be helpful in establishing the cause, but it is not justified.

\section{References}

1 Hepper NG, Spencer-Payne W, Sheps SG, Hyatt RE. Unilateral hypoperfusion of the lung and carcinoid syndrome due to bronchial carcinoid tumor. Am Rev Respir Dis 1977; 115:351-7.

2 Zellos S. Bronchial adenoma. Thorax 1962; 17: 61-8.

3 Wilkins EW Jr, Darling CR, Soutter L, Sniffen $R$. A continuing clinical survey of adenomas of the trachea and bronchus in a general hospital. J Thorac Cardiovasc Surg 1963; 46:279-91.

4 Batson JF, Gale JW, Hickey RC. Bronchial adenoma: a clinical resumé. Arch Surg 1966; 115: 351.

5 Pieron R, Aigueperse J, Oustrières G, Gros H, Kourilsky R. Tumeur carcinoide de la bronche principale droite traitée par bronchotomie et broncho-anastomose correction des troubles ventilatoires et circulatoires. J Fr Med Chir Thorac 1967; 21:497-515.

6 Chiorazzi N, Weiss HS, Margouleff D, Farber S, Gulotta SJ. Long-term pulmonary blood flow alterations following relief of partial bronchial obstruction. Am J Med 1974; 56:559-64.

7 Cheek RC, Wilson H. Carcinoid tumors. Current Problems in Surgery 1970; 4-30. 
8 Euler US von, Liljestrand G. Observation on the pulmonary arterial blood pressure in the cat. Acta Physiol Scand 1946; 12:301-20.

9 Dirken MNJ, Heemstra $\mathrm{H}$. The adaptation of the lung circulation to the ventilation. $Q J \operatorname{Exp}$ Physiol 1948; 34:213-26.

10 Peters RM, Roos A. Effect of unilateral nitrogen breathing upon pulmonary blood flow. $A m$ $J_{C}$ Physiol 1952; 171:250-5.

11 Rahn H, Bahnson HT. Effect of unilateral hypoxia on gas exchange and calculated pulmonary blood $\propto$ flow in each lung. J Appl Physiol 1953-4; 6: $105-12$. 